\title{
Polarity Inversion in Polar-Nonpolar-Polar Heterostructures
}

\author{
Sunglae Cho \\ Department of Physics, University of Ulsan, Ulsan, 680-749, South Korea
}

Suk J. Youn, Yunki Kim, Antonio DiVenere, George K. L. Wong,* Authur J. Freeman, and J. B. Ketterson ${ }^{\dagger}$

Department of Physics and Astronomy, Northwestern University, Evanston, Illinois 60208

(Received 1 May 2001; published 3 September 2001)

\begin{abstract}
We have observed an epilayer-thickness-dependent polarity inversion for the growth of CdTe on $\mathrm{Sb}(\mathrm{Bi}) / \mathrm{CdTe}(111) B$. For films with $\mathrm{Sb}(\mathrm{Bi})$ thicknesses of less than $40 \AA$ (15 $\AA$ ), the CdTe layer shows a $B$ (Te-terminated) face, but it switches to an $A$ (Cd-terminated) face for thicker layers. On the other hand, a CdTe layer grown on $\mathrm{Bi}(\mathrm{Sb}) / \mathrm{CdTe}(111) A$ always shows the $A$ face regardless of $\mathrm{Sb}$ or $\mathrm{Bi}$ layer thicknesses. In order to address the observations we have performed $a b$ initio calculations, which suggest that the polarity of a polar material on a nonpolar one results from the binding energy difference between the two possible surface configurations.
\end{abstract}

DOI: 10.1103/PhysRevLett.87.126403

PACS numbers: 71.15.Nc, 73.20.-r, 77.22.Ej

The zinc-blende (wurtzite) crystal structure is noncentrosymmetric, leading to nonequivalent $(111)[(00.1)]$ vs $(\overline{1} \overline{1} \overline{1})[(00 . \overline{1})]$ planes, which is referred to as surface polarity. For example, the $A$ face for $\mathrm{CdTe}(111)$ is terminated by $\mathrm{Cd}$ atoms, while the $B$ face is terminated by Te. The chemical and physical properties of various epilayers strongly depend on the surface configuration [1-21]. For example, Ga-terminated GaN films show atomically flat surfaces, whereas $\mathrm{N}$-terminated epilayers generate a rough surface morphology with high defect densities [1-17]. In $\mathrm{CdTe}(111)$, the Te-terminated face shows a smooth surface, but the Cd-terminated face is rough [20]. On the other hand, epitaxial growth of a nonpolar material on polar surfaces shows interesting differences compared to growth on nonpolar surfaces. For example, the growth mode [22-25] and surface reconstruction are quite different depending upon whether growth was started on the $A$ or $B$ surfaces. $\alpha$-Sn thin films grow with a biatomic layer-by-layer mode on $\operatorname{InSb}(111) A$ and with a more complicated layerlike mode accompanied by $\mathrm{Sb}$ segregation on $\operatorname{InSb}(111) B$ [25]. Bi grows in a layer-by-layer mode on $\mathrm{CdTe}(111) B$, but exhibits 3D island growth on (111)A until $40 \AA$, thereafter coalescing [22-24]. Thus, surface polarity selection and control of the epilayer is a key issue in obtaining high-quality polar thin films with smooth morphology and low defect density.

In this Letter, we report the observation of polarity inversion; the case in point involves the growth of CdTe on $\mathrm{Sb}(\mathrm{Bi}) / \mathrm{CdTe}(111) B$. For films with $\mathrm{Sb}(\mathrm{Bi})$ thicknesses of less than $40 \AA$ (15 $\AA$ ), the CdTe shows a $B$ (Te-terminated) face, whereas above these thicknesses an $A$ (Cd-terminated) face is observed. On the other hand, a CdTe layer grown on $\mathrm{Bi}(\mathrm{Sb}) / \mathrm{CdTe}(111) A$ always shows the $A$ face, regardless of $\mathrm{Sb}$ or Bi layer thicknesses. Based on experimental and first principles band structure data for $\mathrm{CdTe}(111) / \mathrm{Sb}(\mathrm{Bi}) / \mathrm{CdTe}(111)$ systems, we demonstrate that the polarity of a polar material on a nonpolar one is determined from the binding energy difference between the two possible surface configurations. Thus, the polarity of the top polar layer relative to the bottom polar layer can sometimes be flipped by inserting an ultrathin second material.

We used a custom-built molecular beam epitaxy system to deposit the epilayers which is similar to a Varian Model 360; it consists of growth, analysis, and load-lock chambers and is equipped with reflection high-energy electron diffraction (RHEED) and Auger electron spectroscopy as described elsewhere [22,23]. The base pressure of the growth chamber was in the $10^{-10}$ Torr range. The $\mathrm{CdTe}(111) A / B$ substrates were etched in a solution of $2 \%$ bromine in methanol. After thermal annealing at $300{ }^{\circ} \mathrm{C}$ for $30 \mathrm{~min}$, we deposited a $3000 \AA \mathrm{CdTe}$ buffer layer on the CdTe substrate at $250{ }^{\circ} \mathrm{C}$, resulting in the preparation of a smooth surface. The $\mathrm{Sb}$ or Bi layers were deposited at a rate of $\approx 0.3 \AA / \mathrm{s}$ and at a temperature of $150^{\circ} \mathrm{C}$. The crystal structure of $\mathrm{Sb}$ and $\mathrm{Bi}$ is rhombohedral, and the growth direction on $\mathrm{CdTe}(111)$ is parallel to the trigonal axis. The in-plane lattice mismatches of $\mathrm{Sb}(a=4.308 \AA)$ and $\mathrm{Bi}$ $(a=4.546 \AA)$ with $\mathrm{CdTe}(111)(a=4.58 \AA)$ are $5.9 \%$ and $0.7 \%$, respectively, in the hexagonal setting. RHEED has been used to identify the specific surface reconstruction, growth mode of the deposited layers, and the polarity of the as-grown top CdTe films.

The polarity of $\mathrm{CdTe}(111)$ results in a differing growth mode of $\mathrm{Bi}$ on the $A$ and $B$ faces of $\mathrm{CdTe}(111)$ (the $A$ face is terminated by $\mathrm{Cd}$ atoms, while the $B$ face is terminated by Te atoms). Bi on the $A$ face displays 3D-island growth during the first $40 \AA$, thereafter coalescing, while $\mathrm{Bi}$ on the $B$ face grows layer-by-layer [22,23]. These differing behaviors were exploited as an in situ method of determining the polarity of CdTe; the polarities were confirmed with etch pit experiments [using Nakagawa solution $\left(\mathrm{HF} ; \mathrm{H}_{2} \mathrm{O}_{2}: \mathrm{H}_{2} \mathrm{O}=3: 2: 3 v / v\right)$, which yields etch pits on the (111) $A$ faces and untouched $B$ faces], and the known surface reconstruction differences between $A$ and $B$ faces of CdTe (observed by RHEED) [22]. 
In our earlier studies [22-24], we also showed that $\mathrm{CdTe}(111)$ growth on thick (nonpolar) $\mathrm{Sb}$ or $\mathrm{Bi}$ [on $\alpha-\mathrm{Al}_{2} \mathrm{O}_{3}$ or $\mathrm{CdTe}(111) A / B$ substrates] starts with $\mathrm{Te}$ and ends with $\mathrm{Cd}$, resulting in a (111)A surface. If we deposit $\mathrm{Sb}$ or $\mathrm{Bi}$ on $\mathrm{CdTe}(111) B$ followed by $\mathrm{CdTe}$, the $\mathrm{Te}$ planes in both CdTe layers face the $\mathrm{Sb}$ or Bi layers, provided that the intermediate layer is not too thin. It is of interest to examine the polarity of the CdTe overlayer as the intermediate semimetal layer thickness goes to zero. Clearly, the polarity of the CdTe overlayer must change from $A$ to $B$ in the limit of zero thickness. We have investigated the polarity of the CdTe overlayer for varying intermediate semimetal layer thickness.

Figures 1(a) and 1(b) show the (110) azimuth RHEED patterns of a $3000 \AA \mathrm{CdTe}(111) B$ buffer layer and after a $5 \AA \mathrm{Sb}$ layer subsequently grown. The $\mathrm{CdTe}(111) B$ face has a $2 \sqrt{3} \times 2 \sqrt{3}-R 30^{\circ}$ surface reconstruction. $\mathrm{Sb}$ on the $\operatorname{CdTe}(111) B$ showed no surface reconstruction and streaky patterns, representing layer-by-layer growth of
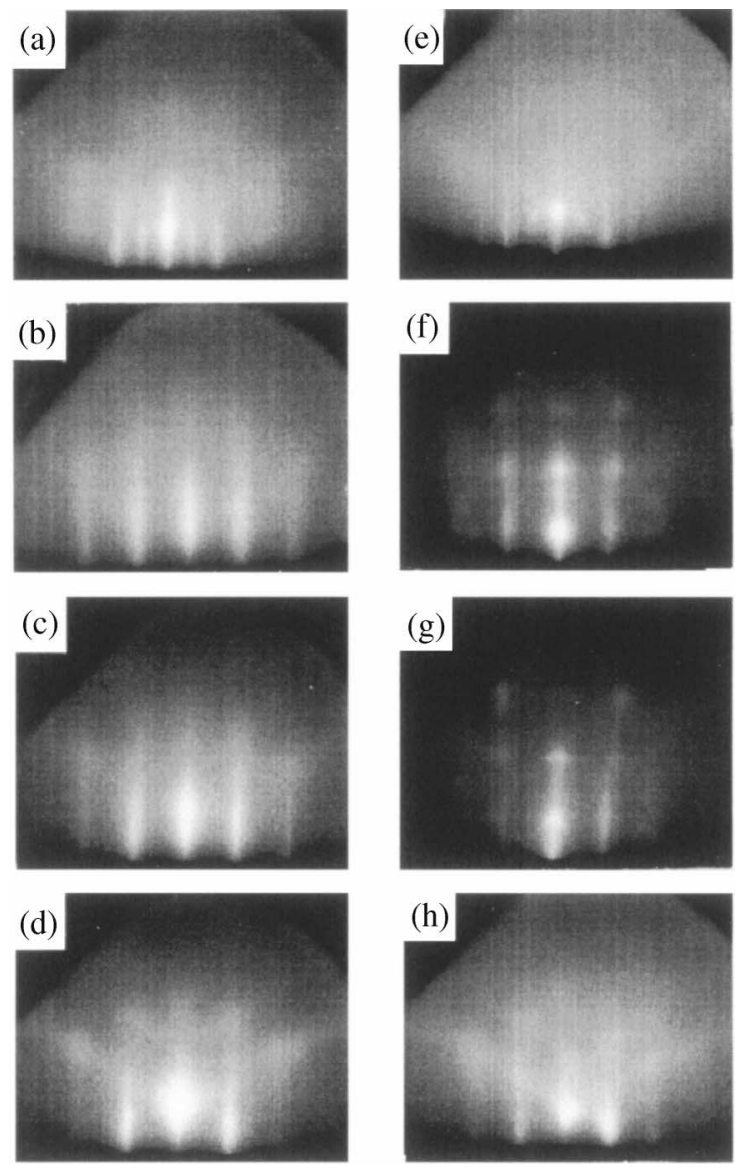

FIG. 1. RHEED patterns of (a) of $\mathrm{CdTe}(111) B$ buffer (1110) azimuth; (b) $5 \AA \mathrm{Sb}$ on $\operatorname{CdTe}(111) B$; (c) $150 \AA \mathrm{CdTe}$ grown on $5 \AA \mathrm{Sb} / \mathrm{CdTe}(111) B$; (d) $15 \AA \mathrm{Bi}$ grown on $150 \AA$ $\mathrm{CdTe} / 5 \AA \mathrm{Sb} / \mathrm{CdTe}(111) B$; (e) $15 \AA \mathrm{Bi}$ grown on $150 \AA \mathrm{CdTe} /$ $35 \AA \mathrm{Sb} / \mathrm{CdTe}(111) B$; (f) $15 \AA \mathrm{Bi}$ grown on $150 \AA \mathrm{CdTe} / 50 \AA$ $\mathrm{Sb} / \mathrm{CdTe}(111) B$; (g) $15 \AA \mathrm{Bi}$ grown on $150 \AA \mathrm{CdTe} / 5 \AA$ $\mathrm{Sb} / \mathrm{CdTe}(111) A$; and (h) $15 \AA \mathrm{Bi}$ grown on $150 \AA \mathrm{CdTe} /$ $50 \AA \mathrm{Sb} / \mathrm{CdTe}(111) A$.
Sb. Figure 1(c) shows the RHEED pattern of $150 \AA$ of $\mathrm{CdTe}(111)$ on $5 \AA \mathrm{Sb} / \mathrm{CdTe}(111) B$. The patterns resulting from depositing a $15 \AA \mathrm{Bi}$ layer on $150 \AA \mathrm{CdTe} / 5 \AA$ $\mathrm{Sb} / \mathrm{CdTe}(111) B$ is streaky, as shown in Fig. 1(d), indicating a $B$ face for the top CdTe layer. When the intermediate Sb layer thickness was increased to $35 \AA$, the $B$ face was also observed [Fig. 1(e)]. However, the RHEED pattern of $\mathrm{Bi}$ on $150 \AA \mathrm{CdTe} / 50 \AA \mathrm{Sb} / \mathrm{CdTe}(111) B$ shows a spotty pattern [Fig. 1(f)], representing a 3D-island growth of Bi and an $A$ face for the top CdTe layer. The Sb critical thickness for the polarity inversion from the $B$ to the $A$ of $\mathrm{CdTe}$ on $\mathrm{Sb} / \mathrm{CdTe}(111) B$ is therefore around $40 \AA$. On the other hand, in the case of $\operatorname{CdTe}(111) A$ substrates, the polarity of the top CdTe layer always remains $A$ face, regardless of the intermediate $\mathrm{Sb}$ layer thickness, as shown in Figs. 1(g) and 1(h). Note Sb grows layer-by-layer on either the $A$ or the $B$ face of CdTe(111).

When $\mathrm{Bi}$ is substituted for $\mathrm{Sb}$, we have observed similar results except the critical thickness $(\sim 15 \AA)$ for the polarity inversion is smaller. Results similar to ours have been observed by Omi et al. [25]: InSb on $\alpha-\operatorname{Sn}(111) / \operatorname{InSb}(111) B$ changes its surface polarity with $\alpha$-Sn thickness. Below $20 \AA$ of $\alpha$-Sn, the InSb exhibits the $B$ face (Sb-terminated), whereas above this thickness InSb becomes $A$ face (In-terminated). InSb on $\alpha$-Sn(111)/InSb(111) $A$ always shows an $A$ face. In Table I we summarized our results as well as the literature result for the $\alpha$-Sn/InSb case. The polarity of $\mathrm{CdTe}$ grown on $\mathrm{Sb}$ or $\mathrm{Bi}$ is $A$ face, independent of the polarity of the $\mathrm{CdTe}(111)$ substrate, except when the Bi or Sb thickness falls below the critical thickness. Below this critical thickness, the polarity of CdTe grown on $\mathrm{Sb}(\mathrm{Bi}) / \mathrm{CdTe}(111) B$ changes to $A$ face.

We now address what causes this polarity inversion. In order to explore why the $\mathrm{Te}$ face attaches to nonpolar $\mathrm{Sb}$ or $\mathrm{Bi}$, we performed an electronic structure calculation using the full-potential linearized augmented plane wave method which does not impose any shape approximation on the potential. This particular code is adapted to calculate the electronic properties of a "slab" of a specified number of layers and stacking sequences. The total energies for the two cases in which $\mathrm{Te}$ is adjacent to $\mathrm{Sb}$ and $\mathrm{Cd}$ is adjacent to $\mathrm{Sb}$ were calculated for a $\mathrm{Cd}-\mathrm{Te}(\mathrm{Te}-\mathrm{Cd})$ layer on four $\mathrm{Sb}$ layers. We assumed the bulk electronic structure for the fourth $\mathrm{Sb}$ layer from the interface and used the in-plane

TABLE I. Summary of the polarity of the top CdTe and InSb [25] layers on polar substrates with intermediate layers.

\begin{tabular}{ccc}
\hline \hline Substrate & Intermediate layer & Polarity of top layer \\
\hline $\operatorname{CdTe}(111) B$ & $>40 \AA \mathrm{Sb}$ or $15 \AA \mathrm{Bi}$ & $\mathrm{CdTe}(111) A$ \\
& $<40 \AA \mathrm{Sb}$ or $15 \AA \mathrm{Bi}$ & $\mathrm{CdTe}(111) B$ \\
$\mathrm{CdTe}(111) A$ & $\mathrm{Sb}$ or $\mathrm{Bi}$ & $\mathrm{CdTe}(111) A$ \\
$\operatorname{InSb}(111) B$ & $>20 \AA \alpha-\mathrm{Sn}$ & $\operatorname{InSb}(111) A$ \\
& $<20 \AA \alpha-\mathrm{Sn}$ & $\operatorname{InSb}(111) B$ \\
$\operatorname{InSb}(111) A$ & $\alpha-\mathrm{Sn}$ & $\operatorname{InSb}(111) A$ \\
\hline \hline
\end{tabular}


lattice constant of bulk Sb. Relaxation was included along the (111) direction by means of atomic force calculations. The calculated total binding energy of an $\mathrm{Sb}$-Te pair is $16 \mathrm{meV}$ lower than that for an Sb-Cd pair [26]. This result implies that a Sb-Te pair is energetically more favorable than a Sb-Cd pair, resulting in the initial Te layer growth on $\mathrm{Sb}$ or $\mathrm{Bi}$ and ending with a $\mathrm{Cd}$ layer (i.e., $A$ face). Therefore, it is not surprising that a CdTe layer grown on thick $\mathrm{Sb}(\mathrm{Bi}) / \mathrm{CdTe}(111) B$ exhibits the $A$ face. In this case, the Te atom layers in both CdTe layers (top layer and substrate) are facing the $\mathrm{Sb}$ or $\mathrm{Bi}$ layer.

In order to further investigate the above system, we calculated the charge density and charge transfer near the $\mathrm{Sb}-\mathrm{Te}$ interface as shown in Figs. 2(a) and 2(b), respectively. We can clearly see the chemical bonding between the CdTe and Sb layers. A charge transfer plot shows that more electrons accumulate near the $\mathrm{Te}$ site than the $\mathrm{Cd}$ site. This indicates that, while the bonding is mostly covalent, there is a small ionic character between $\mathrm{Cd}$ and $\mathrm{Te}$ atoms, which possibly results in a surface polarization.

As mentioned earlier, the initial Te layer growth on thick $\mathrm{Sb}(\mathrm{Bi})$ is due to stronger $\mathrm{Sb}(\mathrm{Bi})$-Te bonding as opposed to $\mathrm{Sb}(\mathrm{Bi})-\mathrm{Cd}$ bonding, resulting in $A$-face termination [Fig. 3(a)]. In this case, the Te layers in both CdTe layers face the $\mathrm{Sb}(\mathrm{Bi})$ layers. The polarity inversion on ultrathin $\mathrm{Sb}(\mathrm{Bi}) / \mathrm{CdTe}(111) B$ indicates the presence of another mechanism which overcomes the binding energy difference between $\mathrm{Sb}(\mathrm{Bi})-\mathrm{Te}$ and $\mathrm{Sb}(\mathrm{Bi})-\mathrm{Cd}$. Based on the facts that the polarity of the CdTe overlayer on $\mathrm{CdTe}(111) B$ must change from $A$ to $B$ in the limit of zero intermediate layer thickness, and that a small ionic character between $\mathrm{Cd}$ and $\mathrm{Te}$ atoms possibly induces a surface polarization, we suggest that this second mechanism may involve the
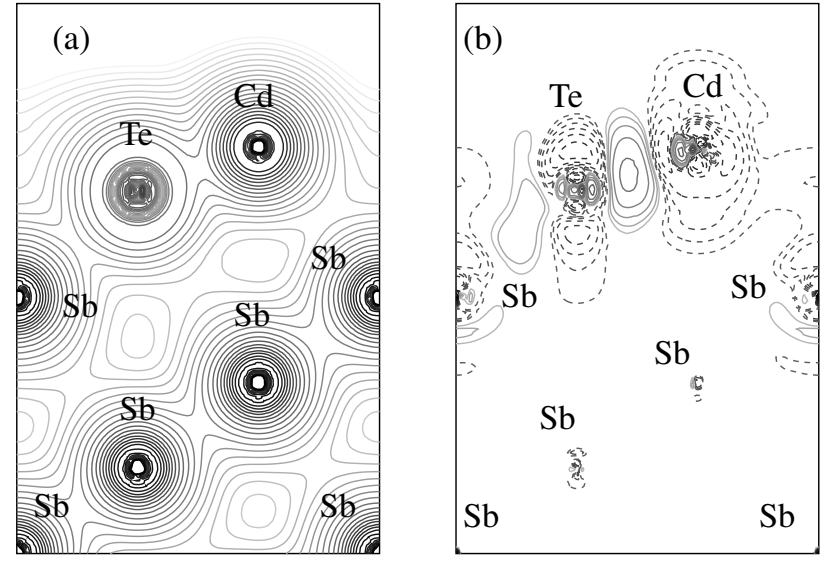

FIG. 2. (a) The charge density and (b) the charge transfer for a CdTe layer on $\mathrm{Sb}$ near the $\mathrm{Te}-\mathrm{Sb}$ interface in the (110) plane. In (a), we can see chemical bonding at the CdTe and Sb interfaces. The charge transfer is defined by $\Delta \rho=\rho_{\mathrm{CdTe} / \mathrm{Sb}}-\rho_{\mathrm{Cd}}-\rho_{\mathrm{Te}}-\rho_{\mathrm{Sb}}$ where $\rho_{\mathrm{CdTe} / \mathrm{Sb}}$, $\rho_{\mathrm{Cd}}, \rho_{\mathrm{Te}}$, and $\rho_{\mathrm{Sb}}$ are the charge densities of $\mathrm{CdTe} / \mathrm{Sb}$, the $\mathrm{Cd}$ monolayer, the Te monolayer, and the Sb layers, respectively. In (b), the solid and dotted lines represent a charge accumulation and depletion, respectively. additional electrostatic interaction arising from the surface induced polarization, as illustrated in Fig. 3, whose direction is downward and upward for the $B$ and the $A$ faces of the CdTe substrate, respectively. In the case of the $\mathrm{CdTe}(111) A /$ thick $\mathrm{Sb}(\mathrm{Bi}) / \mathrm{CdTe}(111) B$ system [Fig. 3(a)], the direction of the two polarizations is opposite, which may result in a repulsive force. However, when the distance between the two polar layers is large, this interaction will be negligible due to electrostatic screening. As the $\mathrm{Sb}$ or Bi layer thickness decreases, the interaction will increase, and eventually the polarity inverts in order to minimize this electrostatic interaction energy.

Thus, polarity inversion is expected when

$$
E_{p-p} \geq \Delta E_{b},
$$

where $E_{p-p}$ is the electrostatic interaction energy between the top layer and the substrate, and $\Delta E_{b}$ is the binding energy difference between $\mathrm{Sb}(\mathrm{Bi})-\mathrm{Te}$ and $\mathrm{Sb}(\mathrm{Bi})$-Cd interfaces. The differing critical thickness for $\mathrm{Sb}$ and $\mathrm{Bi}$ intermediate layers may be correlated with a binding energy difference, different dielectric constants, and the strength of conduction electron screening. Similarly, we can apply this model to an $A$-face substrate. In the case of a $\mathrm{CdTe}(111) A$ substrate with both thin and thick $\mathrm{Sb}(\mathrm{Bi})$ layers, the two polarizations are parallel [Fig. 3(b)]; i.e., the interaction energy is lower, and the above relation is not satisfied. Therefore, as the intermediate $\mathrm{Sb}(\mathrm{Bi})$ layer thickness decreases the polarity does not change, as observed. This model may also apply to the $\mathrm{InSb} / \alpha$-Sn/InSb system.

In conclusion, we have observed a polarity inversion of the CdTe top layer on $\mathrm{Sb}(\mathrm{Bi}) / \mathrm{CdTe}(111) B$ with ultrathin $\mathrm{Sb}$ or Bi layer thicknesses: For films with $\mathrm{Sb}(\mathrm{Bi})$ thicknesses of less than $40 \AA$ ( $15 \AA)$, the CdTe shows a $B$ (Te-terminated) face, whereas above these thicknesses an $A$
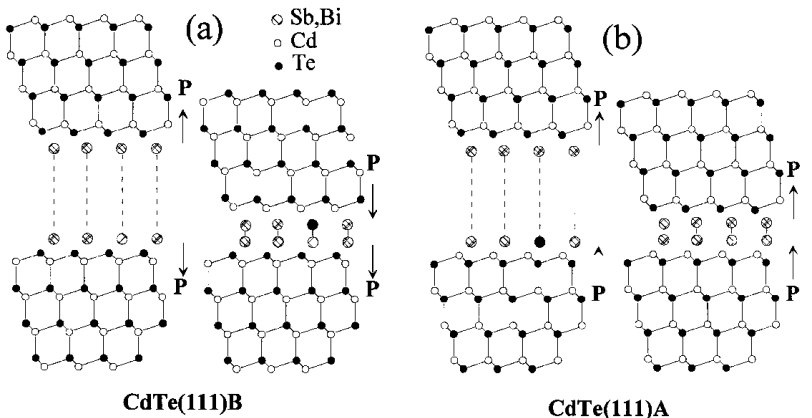

FIG. 3. Schematic views of the polarity properties of $\mathrm{CdTe}(111)$ grown on (a) $\mathrm{CdTe}(111) B$ and (b) $\operatorname{CdTe}(111) A$ with $\mathrm{Sb}(\mathrm{Bi})$ intermediate layers. $P$ represents the surface induced polarization. For a thick $\mathrm{Sb}$ or Bi layer on $\operatorname{CdTe}(111) B$, the polarity of a CdTe overlayer is $A$; i.e., the two surface polarizations are opposite. Below the critical thickness of $\mathrm{Sb}(\mathrm{Bi})$ on $\operatorname{CdTe}(111) B$, the polarity of the $\mathrm{CdTe}$ overlayer changes from $A$ to $B$ due to the repulsive electrostatic interaction. The polarity of a CdTe overlayer on $\mathrm{Sb}(\mathrm{Bi}) / \mathrm{CdTe}(111) A$ is $A$ regardless of the thickness of the semimetal layer because the two polarizations are always parallel. 
(Cd-terminated) face is observed. Our results indicate that the polarity of a polar material on a nonpolar one is determined by the binding energy difference of the two different surface configurations and can be controlled by modifying substrate surface states, e.g., by inserting an ultrathin second species. This "polarity engineering" should prove to be very useful in fundamental surface-related studies as well as in the device-design arena: e.g., for fabricating phase matched waveguides for second harmonic generation [27] or microwave/infrared multilayer acoustic transducers [28].

This work was supported by DARPA under Grant No. DAAG55-97-1-0130 and by the Basic Research Program of the Korea Science and Engineering Foundation under Grant No. 2000-1-11400-002-3. Use was made of central facilities supported by the National Science Foundation, at the Materials Research Center of Northwestern University, under Award No. DMR-9120521.

*Permanent address: Physics Department, HKUST, Clearwater Bay, Kowloon, Hong Kong.

${ }^{\dagger}$ Also at Department of Electrical and Computer Engineering.

[1] E.S. Hellman, D. N.E. Buchanan, D. Wiesmann, and I. Brener, MRS Internet J. Nitride Semicond. Res. 1, U117 (1996).

[2] M. Sumiya et al., MRS Internet J. Nitride Semicond. Res. 4, U462 (1999).

[3] R. Held et al., J. Appl. Phys. 85, 7697 (1999).

[4] Q. Xue et al., Phys. Rev. B 59, 12604 (1999).

[5] K. Rapcewicz, M. B. Nardelli, and J. Bernhol, Phys. Rev. B 56, R12725 (1997).

[6] V. Ramachandran et al., Appl. Phys. Lett. 75, 808 (1999).
[7] E. C. Piquette, P. M. Bridger, Z.Z. Bandic, and T.C. McGill, J. Vac. Sci. Technol. B 17, 1241 (1999).

[8] M. Seelmann-Eggebert et al., Appl. Phys. Lett. 71, 2635 (1997).

[9] F. Hamdani et al., Appl. Phys. Lett. 71, 3111 (1997).

[10] T. Sasaki and T. Matsuoka, J. Appl. Phys. 64, 4531 (1988).

[11] P. Kung et al., J. Appl. Phys. 75, 4515 (1994).

[12] F. A. Ponce et al., Appl. Phys. Lett. 69, 337 (1996).

[13] A. Kazimirov et al., J. Appl. Phys. 84, 1703 (1998).

[14] B. Daudin, J. L. Rouvière, and M. Arley, Appl. Phys. Lett. 69, 2480 (1996).

[15] A. R. Smith et al., Appl. Phys. Lett. 72, 2114 (1998).

[16] J. L. Rouviere, J. L. Weyher, M. Seelmann-Eggebert, and S. Porowski, Appl. Phys. Lett. 73, 668 (1998).

[17] M. Sumiya et al., Appl. Phys. Lett. 75, 674 (1999).

[18] G. Cohen-Solal, F. Bailly, and M. Barbé, Appl. Phys. Lett. 49, 1519 (1986).

[19] T. Ohnishi et al., Appl. Phys. Lett. 72, 824 (1998).

[20] I. Sugiyama and Y. Nishijima, Appl. Phys. Lett. 66, 2798 (1995).

[21] L. Li, C. Tindall, Y. Hasegawa, and T. Sakurai, Appl. Phys. Lett. 71, 2776 (1997).

[22] A. DiVenere et al., Appl. Phys. Lett. 62, 2640 (1993).

[23] S. Cho et al., Phys. Rev. B 58, 2324 (1998).

[24] S. Cho, A. DiVenere, G. K. Wong, and J. B. Ketterson (unpublished).

[25] H. Omi, H. Saito, and T. Osaka, Phys. Rev. Lett. 72, 2596 (1994).

[26] We calculated for the stacking sequence of $A_{\mathrm{Sb}}-B_{\mathrm{Sb}}-C_{\mathrm{Sb}}-A_{\mathrm{Cd} / \mathrm{Te}}-B_{\mathrm{Te} / \mathrm{Cd}}$. For the first $\mathrm{Cd} / \mathrm{Te}$ atom on the top of the $\mathrm{Sb}$ atom ( $C$ position), the total energy is $0.2 \mathrm{eV}$ higher than that of the hollow position $(A)$.

[27] Y. Azumai, M. Kishimoto, I. Seo, and H. Sato, IEEE J. Quantum Electron. 30, 1924 (1994).

[28] H. K. Wong, G. K. Wong, and J. B. Ketterson, J. Appl. Phys. 53, 6834 (1982). 